Figure 2: 100 cells were counted based on nucleus staining and within these cells the number of RV positives was counted. The procedure was repeated on the same slide in two randomly selected areas.

RV positive cells of 100

\begin{tabular}{|c|c|c|c|c|c|c|c|}
\hline & & $\begin{array}{l}\text { Day1 } \\
\text { count } 1\end{array}$ & count2 & mean & count 1 & count 2 & mean \\
\hline \multirow[t]{11}{*}{ controls } & & & & RV & & & OM 10 \\
\hline & 1 & 81 & 89 & 85.0 & 51.0 & 58.0 & 54.5 \\
\hline & 2 & 75 & 68 & 71.5 & 53.0 & 50.0 & 51.5 \\
\hline & 3 & 93 & 99 & 96.0 & 89.0 & 81.0 & 85.0 \\
\hline & 4 & 79 & 86 & 82.5 & 67.0 & 76.0 & 71.5 \\
\hline & 5 & 76 & 75 & 75.5 & 65.0 & 70.0 & 67.5 \\
\hline & 6 & 69 & 57 & 63.0 & 45.0 & 50.0 & 47.5 \\
\hline & 7 & 95 & 94 & 94.5 & 51.0 & 55.0 & 53.0 \\
\hline & 8 & 92 & 99 & 95.5 & 47.0 & 50.0 & 48.5 \\
\hline & 9 & 63 & 79 & 71.0 & 56.0 & 68.0 & 62.0 \\
\hline & 10 & 64 & 54 & 59.0 & 55.0 & 52.0 & 53.5 \\
\hline mean & & & & 79.35 & & & 59.45 \\
\hline \multirow[t]{2}{*}{ sem } & & & & 3.78333333 & & & 3.21333333 \\
\hline & & & & 1 & & & \\
\hline \multirow[t]{11}{*}{ asthma } & & & & $\mathrm{RV}$ & & & OM 10 \\
\hline & 1 & 91 & 96 & 93.5 & 44.0 & 52.0 & 48.0 \\
\hline & 2 & 88 & 83 & 85.5 & 35.0 & 28.0 & 31.5 \\
\hline & 3 & 66 & 79 & 72.5 & 64.0 & 57.0 & 60.5 \\
\hline & 4 & 60 & 65 & 62.5 & 45.0 & 40.0 & 42.5 \\
\hline & 5 & 70 & 78 & 74.0 & 31.0 & 34.0 & 32.5 \\
\hline & 6 & 69 & 82 & 75.5 & 47.0 & 45.0 & 46.0 \\
\hline & 7 & 67 & 60 & 63.5 & 56.0 & 57.0 & 56.5 \\
\hline & 8 & 56 & 49 & 52.5 & 39.0 & 48.0 & 43.5 \\
\hline & 9 & 54 & 56 & 55.0 & 48.0 & 53.0 & 50.5 \\
\hline & 10 & 89 & 80 & 84.5 & 54.0 & 45.0 & 49.5 \\
\hline mean & & & & 71.9 & & & 46.1 \\
\hline sem & & & & 3.60666667 & & & 2.3 \\
\hline \multirow[t]{9}{*}{ COPD } & & & & $\mathrm{RV}$ & & & OM 10 \\
\hline & 1 & 89 & 84 & 86.5 & 66.0 & 63.0 & 66.0 \\
\hline & 2 & 54 & 56 & 55.0 & 47.0 & 56.0 & 47.0 \\
\hline & 3 & 78 & 71 & 74.5 & 55.0 & 52.0 & 55.0 \\
\hline & 4 & 85 & 76 & 80.5 & 66.0 & 71.0 & 66.0 \\
\hline & 5 & 81 & 85 & 83.0 & 54.0 & 63.0 & 54.0 \\
\hline & 6 & 64 & 65 & 64.5 & 42.0 & 44.0 & 42.0 \\
\hline & 7 & 86 & 71 & 78.5 & 30.0 & 33.0 & 30.0 \\
\hline & 8 & 80 & 86 & 83.0 & 59.0 & 68.0 & 59.0 \\
\hline mean & & & & 75.6875 & & & 52.375 \\
\hline sem & & & & 3.1241126 & & & 3.60247388 \\
\hline
\end{tabular}


Figure 3: Epithelial cell survival was manually counted in a modified Neugebauer chamber slide, after trypsin treatment and single cell suspension.

\begin{tabular}{lrrrr} 
dead cells (\%) & \multicolumn{2}{l}{ RV } \\
day & \multicolumn{2}{c}{ Counts } \\
control & 1 & 2 & 3 & 4 \\
c1 & 67 & 59 & 53 & 37 \\
c2 & 73 & 62 & 47 & 26 \\
c3 & 59 & 55 & 41 & 31 \\
c4 & 64 & 51 & 66 & 38 \\
c5 & 58 & 49 & 44 & 42 \\
c6 & 69 & 67 & 37 & 29 \\
c7 & 61 & 54 & 49 & 37 \\
c8 & 58 & 63 & 44 & 28 \\
c9 & 75 & 35 & 21 & 0 \\
c10 & 64 & 59 & 30 & 19 \\
mean & 64.8 & 55.4 & 43.2 & 28.7 \\
sem & 1.931033 & 2.868217 & 3.909533 & 3.847221
\end{tabular}

$\begin{array}{lrrrr}\text { asthma } & 1 & 2 & 3 & 4 \\ \text { a1 } & 65 & 43 & 52 & 38 \\ \text { a2 } & 58 & 59 & 41 & 32 \\ \text { a3 } & 64 & 61 & 53 & 29 \\ \text { a4 } & 61 & 74 & 38 & 21 \\ \text { a5 } & 59 & 48 & 32 & 14 \\ \text { a6 } & 47 & 44 & 32 & 25 \\ \text { a7 } & 49 & 47 & 41 & 27 \\ \text { a8 } & 53 & 43 & 28 & 31 \\ \text { a9 } & 51 & 48 & 32 & 31 \\ \text { a10 } & 57 & 55 & 47 & 41 \\ \text { mean } & 56.4 & 52.2 & 39.6 & 28.9 \\ \text { sem } & 1.961859 & 3.172101 & 2.793644 & 2.474088\end{array}$

$\begin{array}{lrrrr}\text { asthma } & 1 & 2 & 3 & \\ \text { a1 } & 64 & 62 & 41 & 2 \\ \text { a2 } & 47 & 69 & 41 & 3 \\ \text { a3 } & 58 & 57 & 31 & 2 \\ \text { a4 } & 64 & 63 & 54 & 3 \\ \text { a5 } & 59 & 47 & 41 & 4 \\ \text { a6 } & 51 & 43 & 38 & 2 \\ \text { a7 } & 69 & 58 & 49 & 4 \\ \text { a8 } & 63 & 61 & 46 & 2 \\ \text { a9 } & 60 & 55 & 42 & 2 \\ \text { a10 } & 74 & 75 & 69 & 7 \\ \text { mean } & 60.9 & 59 & 45.2 & 37 . \\ \text { sem } & 2.505328 & 2.992583 & 3.292416 & 4.70094\end{array}$

COPD

$\begin{array}{lrrrrlrrrr}\text { SM1 } & 54 & 43 & 39 & 21 & \text { SM1 } & 64 & 58 & 42 & 2 \\ \text { sm2 } & 58 & 49 & 43 & 25 & \text { sm2 } & 62 & 61 & 58 & 4 \\ \text { sm3 } & 61 & 57 & 41 & 37 & \text { sm3 } & 59 & 52 & 46 & 3 \\ \text { sm4 } & 66 & 63 & 64 & 24 & \text { sm4 } & 63 & 49 & 44 & 2 \\ \text { sm5 } & 73 & 64 & 42 & 19 & \text { sm5 } & 74 & 63 & 52 & 3 \\ \text { sm6 } & 53 & 48 & 42 & 36 & \text { sm6 } & 51 & 42 & 33 & 1 \\ \text { sm7 } & 59 & 49 & 27 & 21 & \text { sm7 } & 67 & 63 & 54 & 2 \\ \text { mean } & 60.57143 & 53.28571 & 42.57143 & 26.14286 & \text { mean } & 62.85714 & 55.42857 & 47 & 3 \\ \text { sem } & 2.212518 & 2.560692 & 3.458599 & 2.326862 & \text { sem } & 2.231805 & 2.535463 & 2.664583 & 2.79284\end{array}$


OM .5

day

$\begin{array}{lrrrr}\text { control } & 1 & 2 & 3 & 4 \\ \text { c1 } & 56 & 58 & 53 & 42 \\ \text { c2 } & 74 & 63 & 55 & 39 \\ \text { c3 } & 59 & 51 & 54 & 38 \\ \text { c4 } & 63 & 48 & 44 & 23 \\ \text { c5 } & 55 & 51 & 47 & 31 \\ \text { c6 } & 76 & 71 & 68 & 63 \\ \text { c7 } & 63 & 59 & 45 & 32 \\ \text { c8 } & 86 & 76 & 71 & 54 \\ \text { c9 } & 76 & 53 & 41 & 17 \\ \text { c10 } & 61 & 57 & 36 & 26 \\ \text { mean } & 66.9 & 58.7 & 51.4 & 36.5 \\ \text { sem } & 3.281091 & 2.863758 & 3.56277 & 4.435338\end{array}$

$\begin{array}{lrrrr}\text { asthma } & 1 & 2 & 3 & 4 \\ \text { a1 } & 75 & 63 & 51 & 38 \\ \text { a2 } & 96 & 85 & 76 & 42 \\ \text { a3 } & 68 & 51 & 44 & 27 \\ \text { a4 } & 66 & 61 & 59 & 32 \\ \text { a5 } & 61 & 58 & 59 & 43 \\ \text { a6 } & 59 & 53 & 46 & 38 \\ \text { a7 } & 86 & 75 & 61 & 41 \\ \text { a8 } & 63 & 65 & 48 & 37 \\ \text { a9 } & 65 & 61 & 57 & 43 \\ \text { a10 } & 73 & 64 & 59 & 32 \\ \text { mean } & 71.2 & 63.6 & 56 & 37.3 \\ \text { sem } & 3.723201 & 3.173501 & 2.955221 & 1.713022\end{array}$

COPD

$\begin{array}{lrrrr}\text { SM1 } & 69 & 58 & 53 & 28 \\ \text { sm2 } & 73 & 51 & 49 & 32 \\ \text { sm3 } & 58 & 56 & 48 & 25 \\ \text { sm4 } & 73 & 65 & 58 & 33 \\ \text { sm5 } & 61 & 63 & 51 & 28 \\ \text { sm6 } & 66 & 63 & 65 & 31 \\ \text { sm7 } & 74 & 58 & 62 & 28 \\ \text { mean } & 67.71429 & 59.14286 & 55.14286 & 29.28571 \\ \text { sem } & 1.997618 & 1.543033 & 2.093072 & 0.889087\end{array}$

\begin{tabular}{|c|c|c|c|c|}
\hline OM1 & & & & \\
\hline & day & & & \\
\hline control & 1 & 2 & 3 & \\
\hline c1 & 75 & 62 & 65 & \\
\hline$c 2$ & 58 & 59 & 51 & \\
\hline c3 & 66 & 61 & 58 & \\
\hline c4 & 72 & 68 & 55 & \\
\hline c5 & 85 & 76 & 82 & \\
\hline c6 & 75 & 68 & 58 & \\
\hline c7 & 85 & 64 & 71 & \\
\hline c8 & 68 & 63 & 59 & \\
\hline$c 9$ & 69 & 71 & 65 & \\
\hline c10 & 74 & 61 & 73 & \\
\hline mean & 72.7 & 65.3 & 63.7 & 3 \\
\hline sem & 2.607894 & 1.686877 & 2.985335 & 208 \\
\hline
\end{tabular}

$\begin{array}{lrrr}\text { asthma } & 1 & 2 & 3 \\ \text { a1 } & 69 & 58 & 45 \\ \text { a2 } & 79 & 66 & 53 \\ \text { a3 } & 85 & 75 & 82 \\ \text { a4 } & 79 & 76 & 64 \\ \text { a5 } & 74 & 64 & 54 \\ \text { a6 } & 62 & 54 & 38 \\ \text { a7 } & 75 & 71 & 74 \\ \text { a8 } & 88 & 69 & 73 \\ \text { a9 } & 79 & 68 & 52 \\ \text { a10 } & 85 & 74 & 66\end{array}$

$\begin{array}{llll}\text { mean } & 77.5 & 67.5 & 60.1\end{array}$

sem $\quad \begin{array}{lllll}2.495552 & 2.291288 & 4.420784 & 5.16612\end{array}$

$\begin{array}{lrrrr}\text { SM1 } & 74 & 76 & 64 & 3 \\ \text { sm2 } & 68 & 61 & 48 & 2 \\ \text { sm3 } & 71 & 64 & 41 & 1 \\ \text { sm4 } & 59 & 62 & 58 & 4 \\ \text { sm5 } & 77 & 73 & 65 & 3 \\ \text { sm6 } & 73 & 58 & 55 & 2 \\ \text { sm7 } & 74 & 68 & 68 & 5 \\ \text { mean } & 70.85714 & 66 & 57 & 33.1428 \\ \text { sem } & 1.874643 & 2.089657 & 3.098387 & 4.04733\end{array}$




\begin{tabular}{|c|c|c|c|c|}
\hline \multirow[t]{2}{*}{ OM10 } & \multirow[b]{2}{*}{ day } & \multirow[b]{3}{*}{2} & \multirow[b]{3}{*}{3} & \multirow[b]{3}{*}{4} \\
\hline & & & & \\
\hline control & 1 & & & \\
\hline c1 & 79 & 74 & 75 & 42 \\
\hline c2 & 86 & 84 & 75 & 36 \\
\hline c3 & 89 & 95 & 91 & 66 \\
\hline c4 & 75 & 64 & 69 & 48 \\
\hline c5 & 97 & 85 & 69 & 43 \\
\hline c6 & 83 & 79 & 84 & 39 \\
\hline c7 & 68 & 71 & 65 & 52 \\
\hline c8 & 79 & 75 & 83 & 48 \\
\hline c9 & 94 & 97 & 64 & 61 \\
\hline c10 & 89 & 59 & 53 & 36 \\
\hline mean & 83.9 & 78.3 & 72.8 & 47.1 \\
\hline sem & 2.810496 & 3.896009 & 3.542755 & 3.216105 \\
\hline
\end{tabular}

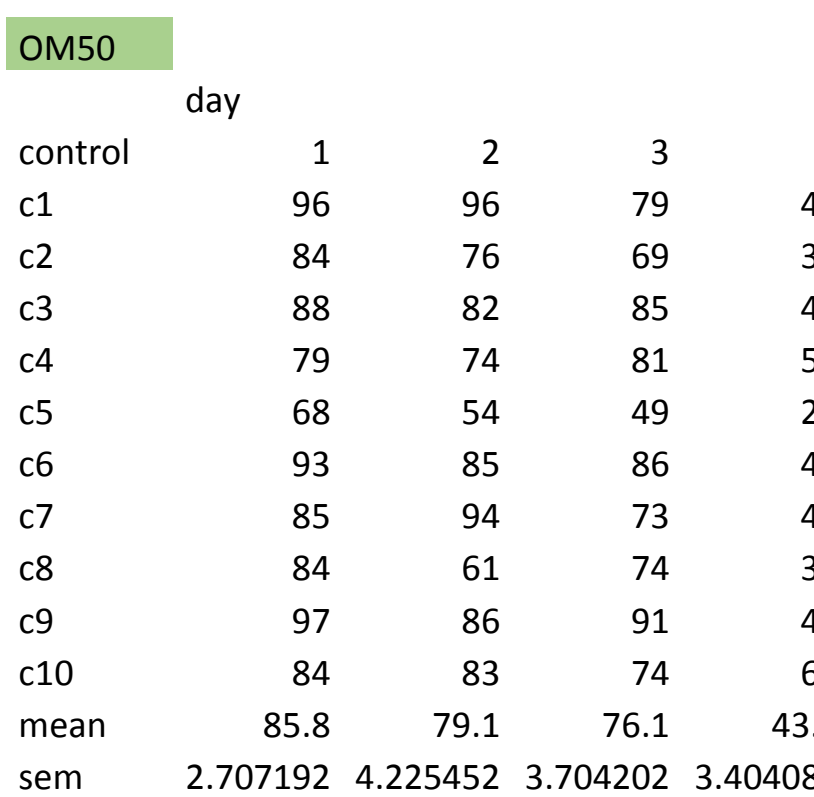

$\begin{array}{lrrrr}\text { asthma } & 1 & 2 & 3 & 4 \\ \text { a1 } & 96 & 95 & 83 & 41 \\ \text { a2 } & 88 & 81 & 75 & 39 \\ \text { a3 } & 81 & 84 & 72 & 48 \\ \text { a4 } & 79 & 64 & 51 & 30 \\ \text { a5 } & 68 & 43 & 49 & 21 \\ \text { a6 } & 77 & 71 & 67 & 55 \\ \text { a7 } & 85 & 78 & 74 & 53 \\ \text { a8 } & 91 & 86 & 74 & 37 \\ \text { a9 } & 69 & 61 & 59 & 43 \\ \text { a10 } & 75 & 72 & 68 & 32 \\ \text { mean } & 80.9 & 73.5 & 67.2 & 39.9 \\ \text { sem } & 2.903829 & 4.702836 & 3.476269 & 3.338163\end{array}$

$\begin{array}{lrrrr}\text { asthma } & 1 & 2 & 3 & \\ \text { a1 } & 86 & 78 & 73 & 2 \\ \text { a2 } & 89 & 74 & 61 & 4 \\ \text { a3 } & 85 & 85 & 53 & 3 \\ \text { a4 } & 79 & 63 & 61 & 2 \\ \text { a5 } & 83 & 74 & 71 & 4 \\ \text { a6 } & 96 & 88 & 75 & 3 \\ \text { a7 } & 74 & 71 & 69 & 5 \\ \text { a8 } & 79 & 64 & 61 & 3 \\ \text { a9 } & 88 & 85 & 81 & 7 \\ \text { a10 } & 93 & 87 & 74 & 4 \\ \text { mean } & 85.2 & 76.9 & 67.9 & 41 . \\ \text { sem } & 2.128119 & 2.922898 & 2.709859 & 4.59516\end{array}$

$\begin{array}{lrrrr}\text { COPD } & & & & \\ \text { SM1 } & 88 & 80 & 65 & 34 \\ \text { sm2 } & 79 & 71 & 54 & 32 \\ \text { sm3 } & 95 & 86 & 75 & 54 \\ \text { sm4 } & 82 & 76 & 69 & 41 \\ \text { sm5 } & 58 & 74 & 53 & 47 \\ \text { sm6 } & 87 & 89 & 74 & 34 \\ \text { sm7 } & 81 & 65 & 73 & 49 \\ \text { mean } & 81.42857 & 77.28571 & 66.14286 & 41.57143 \\ \text { sem } & 3.682649 & 2.656528 & 2.93501 & 2.725541\end{array}$

$\begin{array}{lrrrr}\text { sM1 } & 86 & 76 & 63 & 2 \\ \text { sm2 } & 97 & 93 & 76 & 5 \\ \text { sm3 } & 93 & 70 & 83 & 3 \\ \text { sm4 } & 69 & 74 & 63 & 5 \\ \text { sm5 } & 85 & 74 & 61 & 3 \\ \text { sm6 } & 96 & 58 & 66 & 4 \\ \text { sm7 } & 93 & 86 & 69 & 2 \\ \text { mean } & 88.42857 & 75.85714 & 68.71429 & 40.1428 \\ \text { sem } & 3.076021 & 3.55166 & 2.547641 & 3.44203\end{array}$


Figure 4: Signal transduction of RV and OM-85.

4.A: intra cellular Erk $1 / 2$ MAPK expression (optical density) in minutes ( $T=$ minutes) after addition of compounds. Statistics were calculated as

\begin{tabular}{|c|c|c|c|c|}
\hline otal Erk & $0^{\prime}$ & $15^{\prime}$ & $30^{\prime}$ & $60^{\prime}$ \\
\hline a & 100596 & 116543 & 110694 & 103725 \\
\hline b & 118475 & 109687 & 110583 & 115342 \\
\hline$c$ & 109485 & 110682 & 128574 & 108275 \\
\hline$d$ & 142356 & 132534 & 128574 & 119857 \\
\hline mean & 117728 & 117361.5 & 119606.25 & 111799.75 \\
\hline sem & 7325.13154 & 4379.92348 & 5177.53288 & 3348.48722 \\
\hline phos Erk & $0^{\prime}$ & $15^{\prime}$ & $30^{\prime}$ & $60^{\prime}$ \\
\hline & 86758 & 124138 & 143265 & 156325 \\
\hline & 99706 & 132436 & 154326 & 117482 \\
\hline & 76850 & 176574 & 253418 & 198675 \\
\hline & 99685 & 142367 & 276453 & 228563 \\
\hline eam & 90749.75 & 143878.75 & 206865.5 & 175261.25 \\
\hline $\mathrm{em}$ & 5164.83117 & 9438.30569 & 33526.7301 & 22145.8573 \\
\hline
\end{tabular}

\begin{tabular}{lllll}
$\begin{array}{l}\text { ratio } \\
\text { phos/total }\end{array}$ & \multicolumn{1}{l}{$l$} & \\
Erk & $0^{\prime}$ & $15^{\prime}$ & $30^{\prime}$ & $60^{\prime}$ \\
$\mathrm{a}$ & 0.862439858 & 1.06516908 & 1.29424359 & 1.50711015 \\
$\mathrm{~b}$ & 0.841578392 & 1.20739924 & 1.39556713 & 1.01855352 \\
$\mathrm{c}$ & 0.701922638 & 1.59532715 & 1.97098947 & 1.83491111 \\
$\mathrm{~d}$ & 0.700251482 & 1.07419228 & 2.150147 & 1.90696413 \\
mean & 0.776548093 & 1.23552194 & 1.7027368 & 1.56688473 \\
sem & 0.043567448 & 0.10386682 & 0.20659408 & 0.17554502
\end{tabular}

4.B: intra cellular cAMP levels $(\mathrm{pg} / \mathrm{ml})$ in minutes ( $\mathrm{T}=$ minutes) after addition of compounds. $A=$ asthma; $C=C O P D ; H=$ healthy

\begin{tabular}{|c|c|c|c|c|c|}
\hline \multirow{2}{*}{\multicolumn{2}{|c|}{ Cell lines }} & \multicolumn{4}{|c|}{$\mathrm{OM} 10 \mu \mathrm{g} / \mathrm{ml}$} \\
\hline & & TO & $\mathrm{T} 15$ & T30 & T60 \\
\hline \multirow{4}{*}{ 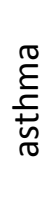 } & A1 & 0.942 & 38.942 & 12.458 & 2.791 \\
\hline & A2 & 0.897 & 93.085 & 22.714 & 21.621 \\
\hline & A3 & 0.947 & 54.858 & 41.679 & 2.341 \\
\hline & mean & 0.929 & 62.295 & 25.617 & 8.918 \\
\hline \multirow{5}{*}{ 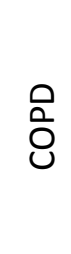 } & $\mathrm{C} 1$ & 0.602 & 193.359 & 19.474 & 6.164 \\
\hline & $\mathrm{C} 2$ & 0.615 & 14.01 & 5.2715 & 16.487 \\
\hline & C3 & 0.704 & 5.973 & 38.202 & 0.859 \\
\hline & $\mathrm{C} 4$ & 0.642 & 58.485 & 72.606 & 0.999 \\
\hline & mean & 0.641 & 67.957 & 33.888 & 6.127 \\
\hline
\end{tabular}




\begin{tabular}{|c|c|c|c|c|}
\hline $\mathrm{H} 1$ & 2.916 & 45.801 & 47.21 & 1.233 \\
\hline $\mathrm{H} 2$ & 1.172 & 49.94 & 90.84 & 1.593 \\
\hline H3 & 1.176 & 95.993 & 158.76 & 1.447 \\
\hline mean & 1.755 & 63.911 & 98.937 & 1.424 \\
\hline MEAN & 1.0613 & 65.0446 & 50.92145 & 5.5535 \\
\hline
\end{tabular}

4.C Expression of total and phosphorylated CREB by western-blotting (optical density)

\begin{tabular}{|c|c|c|c|c|}
\hline total CREB & $0^{\prime}$ & $15^{\prime}$ & $30^{\prime}$ & $60^{\prime}$ \\
\hline $\mathrm{a}$ & 116253 & 109584 & 113256 & 116453 \\
\hline b & 119865 & 116254 & 128475 & 129834 \\
\hline c & 128475 & 127594 & 123847 & 128274 \\
\hline d & 97856 & 132860 & 110968 & 94564 \\
\hline mean & 115612.25 & 121573 & 119136.5 & 117281.25 \\
\hline sem & 5125.78786 & 4996.38923 & 4055.59697 & 6797.00038 \\
\hline phos Creb & $0^{\prime}$ & $15^{\prime}$ & $30^{\prime}$ & $60^{\prime}$ \\
\hline a & 65746 & 112635 & 119856 & 97865 \\
\hline b & 59483 & 110293 & 109567 & 109485 \\
\hline c & 77512 & 101928 & 143527 & 96857 \\
\hline d & 110293 & 123957 & 132437 & 100968 \\
\hline meam & 78258.5 & 112203.25 & 126346.75 & 101293.75 \\
\hline sem & 9247.5636 & 3517.65085 & 6717.61472 & 2364.6102 \\
\hline
\end{tabular}

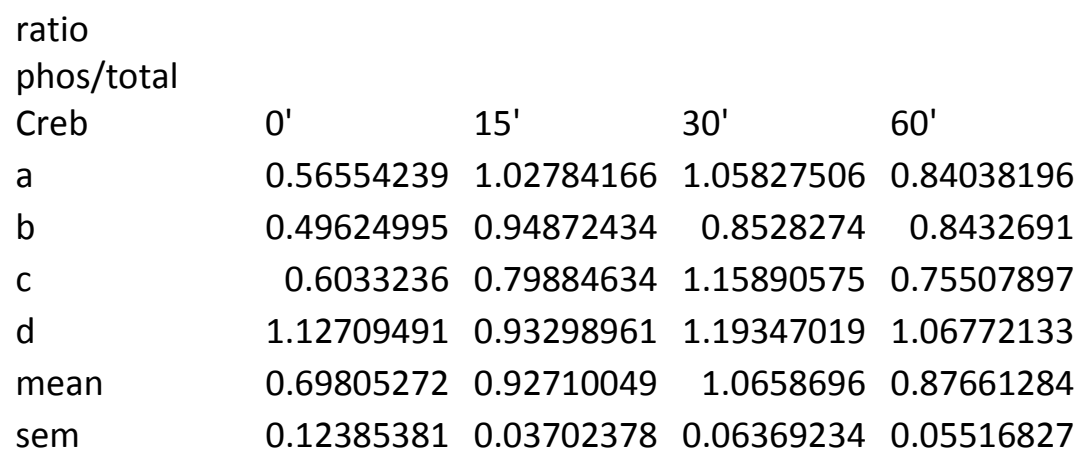

4.D: Effect of signal inhibition on RV replication as described in figure 2.

\begin{tabular}{|c|c|c|c|c|c|c|c|}
\hline & untreated & RV & & RV+DDA (10 uM) & RV + PD98059 & $\begin{array}{l}\text { RV + } \\
\text { SB203580 }\end{array}$ & $\begin{array}{l}\mathrm{RV}+\mathrm{OM}- \\
85\end{array}$ \\
\hline E1 & 0 & 0 & 95 & 95 & 74 & 69 & 43 \\
\hline E2 & 0 & 0 & 52 & 86 & 72 & 85 & 25 \\
\hline E3 & 0 & 0 & 85 & 63 & 69 & 93 & 51 \\
\hline E4 & 0 & 0 & 63 & 76 & 72 & 91 & 37 \\
\hline E5 & 0 & 0 & 78 & 49 & 55 & 76 & 48 \\
\hline mean & 0 & 0 & 74.6 & 73.8 & 68.4 & 82.8 & 40.8 \\
\hline sem & 0 & 0 & 6.84 & 7.12 & 2.68 & 4.12 & 3.92 \\
\hline
\end{tabular}


Figure 5: Role of CqR1 and $\beta$-defensin in RV and OM-85 signalling assessed by western-blot densitometry.

A. C1qR expression determined by Western-blotting (optical density)

\begin{tabular}{|c|c|c|c|c|}
\hline $\begin{array}{l}\text { control } \\
\text { control }\end{array}$ & day 0 & RV day 2 & $\begin{array}{l}\text { OM } 10 \\
\text { ug/ml }\end{array}$ & $\begin{array}{l}R V+O M \\
10 \text { ug }\end{array}$ \\
\hline c1 & 1.098 & 1.132 & 2.657 & 2.473 \\
\hline$c 2$ & 0.905 & 1.218 & 2.186 & 2.196 \\
\hline c3 & 1.125 & 0.968 & 1.978 & 1.978 \\
\hline nean & 1.042667 & 1.106 & 2.273667 & 2.215667 \\
\hline sem & 0.069273 & 0.07333 & 0.200852 & 0.143232 \\
\hline
\end{tabular}

asthma

$\begin{array}{lrrrr}\text { a1 } & 1.165 & 0.978 & 1.978 & 2.318 \\ \text { a2 } & 1.121 & 1.263 & 2.687 & 2.456 \\ \text { a3 } & 0.867 & 1.177 & 1.994 & 1.978 \\ \text { mean } & 1.051 & 1.139333 & 2.219667 & 2.250667 \\ \text { sem } & 0.092873 & 0.084401 & 0.233712 & 0.142034\end{array}$

COPD

$\begin{array}{lrrrr}\text { sm1 } & 1.005 & 0.978 & 2.756 & 2.758 \\ \text { sm2 } & 0.954 & 0.894 & 2.186 & 1.473 \\ \text { sm3 } & 1.102 & 1.123 & 2.574 & 2.175 \\ \text { mean } & 1.020333 & 0.998333 & 2.505333 & 2.135333 \\ \text { sem } & 0.043406 & 0.066884 & 0.168089 & 0.371477\end{array}$

B. Signalling control of C1qR expression determined by Western-blotting (optical density)

$\begin{array}{lccccc}\text { Cell lines } & \text { Control } & \text { OM-85 } & \text { OM+DDA } & \text { OM+SB } & \text { OM+PD } \\ \text { H01 } & 0.783 & 1.251 & 0.824 & 1.159 & 0.686 \\ \text { H02 } & 0.736 & 1.152 & 0.663 & 1.143 & 0.632 \\ \text { H03 } & 0.718 & 0.834 & 0.652 & 0.997 & 0.569 \\ \text { H04 } & 0.476 & 1.135 & 0.345 & 0.75 & 0.742 \\ \text { H05 } & 0.327 & 0.718 & 0.516 & 1.155 & 0.506 \\ \text { H06 } & 0.686 & 1.282 & 0.418 & 0.737 & 0.553 \\ \text { mean } & 0.62 & 1.06 & 0.57 & 0.99 & 0.61 \\ \text { SD } & 0.18 & 0.23 & 0.18 & 0.20 & 0.09 \\ \text { SEM } & 0.08014088 & 0.10351232 & 0.07912859 & 0.08963322 & 0.03964812\end{array}$

C $\beta$-defensin expression determined by western-blotting (optical density)

defensin beta

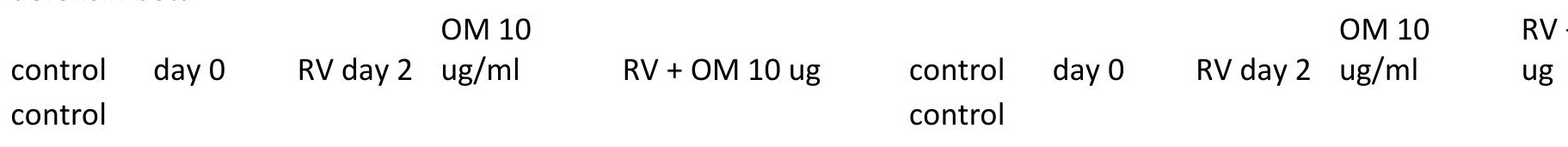




$\begin{array}{lrrrr}\text { h1 } & 95598 & 122330 & 308586 & 317304 \\ \text { h2 } & 108867 & 103443 & 191196 & 169790 \\ \text { h3 } & 108383 & 113613 & 219187 & 266938 \\ \text { h4 } & 124074 & 118069 & 308586 & 293293 \\ \text { h5 } & 95598 & 97438 & 212698 & 308586 \\ \text { h6 } & 107124 & 128529 & 179476 & 257349 \\ \text { mean } & 106607 & & & \end{array}$

asthma

$\begin{array}{lrlll}\text { a1 } & 99412 & 167211 & 223219 & 235620 \\ \text { a2 } & 119436 & 118318 & 290408 & 335133 \\ \text { a3 } & 129500 & 102258 & 323851 & 223321 \\ \text { a4 } & 121673 & 128483 & 312974 & 270079 \\ \text { a5 } & 110186 & 101241 & 222203 & 139461 \\ \text { a6 } & 100936 & 176461 & 291425 & 249647 \\ \text { a7 } & 129601 & 136412 & 261642 & 201060 \\ \text { mean } & 115821 & & & \end{array}$

COPD

$\begin{array}{lrlll}\text { c1 } & 125825 & 154913 & 325385 & 310728 \\ \text { c2 } & 137212 & 105756 & 245335 & 309826 \\ \text { c3 } & 109138 & 112295 & 185693 & 165962 \\ \text { c4 } & 94368 & 132589 & 220869 & 225041 \\ \text { c5 } & 154688 & 136535 & 206663 & 292914 \\ \text { c6 } & 112408 & 178590 & 357969 & 257625 \\ \text { c7 } & 162692 & 149727 & 247703 & 360449 \\ \text { c8 } & 110942 & 207114 & 223012 & 356728 \\ \text { mean } & 125909 & & & \end{array}$

$\begin{array}{llll}\text { h1 } & 0.897 & 1.147 & 2.895 \\ \text { h2 } & 1.021 & 0.970 & 1.793 \\ \text { h3 } & 1.017 & 1.066 & 2.056 \\ \text { h4 } & 1.164 & 1.108 & 2.895 \\ \text { h5 } & 0.897 & 0.914 & 1.995 \\ \text { h6 } & 1.005 & 1.206 & 1.684 \\ \text { mean } & 1.000 & 1.068 & 2.220 \\ \text { sem } & 0.057 & 0.063 & 0.312\end{array}$

asthma

$\begin{array}{llll}\text { a1 } & 0.933 & 1.568 & 2.094 \\ \text { a2 } & 1.120 & 1.110 & 2.724 \\ \text { a3 } & 1.215 & 0.959 & 3.038 \\ \text { a4 } & 1.141 & 1.205 & 2.936 \\ \text { a5 } & 1.034 & 0.950 & 2.084 \\ \text { a6 } & 0.947 & 1.655 & 2.734 \\ \text { a7 } & 1.216 & 1.280 & 2.454 \\ \text { mean } & 1.086 & 1.247 & 2.581 \\ \text { sem } & 0.068 & 0.160 & 0.221\end{array}$

COPD

$\begin{array}{llll}\text { c1 } & 1.180 & 1.453 & 3.052 \\ \text { c2 } & 1.287 & 0.992 & 2.301 \\ \text { c3 } & 1.024 & 1.053 & 1.742 \\ \text { c4 } & 0.885 & 1.244 & 2.072 \\ \text { c5 } & 1.451 & 1.281 & 1.939 \\ \text { c6 } & 1.054 & 1.675 & 3.358 \\ \text { c7 } & 1.526 & 1.404 & 2.324 \\ \text { c8 } & 1.041 & 1.943 & 2.092 \\ \text { mean } & 1.181 & 1.381 & 2.360 \\ \text { sem } & 0.129 & 0.182 & 0.323\end{array}$

D Signalling control $\beta$-defensin expression determined by western-blotting (optical density

$\begin{array}{lccccc}\text { Cell lines } & \text { Control } & \text { OM } & \text { OM+DDA } & \text { OM+SB } & \text { OM+PD } \\ \text { H01 } & 0.4 & 0.595 & 0.755 & 0.548 & 0.5 \\ \text { H02 } & 0.424 & 0.839 & 0.481 & 0.757 & 0.486 \\ \text { H03 } & 0.218 & 0.602 & 0.789 & 0.557 & 0.417 \\ \text { H04 } & 0.434 & 0.601 & 0.492 & 0.532 & 0.465 \\ \text { H05 } & 0.254 & 0.499 & 0.416 & 0.453 & 0.335 \\ \text { H06 } & 0.434 & 0.549 & 0.3 & 0.509 & 0.477 \\ \text { mean } & 0.36 & 0.61 & 0.54 & 0.56 & 0.45 \\ \text { SD } & 0.10 & 0.12 & 0.19 & 0.10 & 0.06 \\ \text { SEM } & 0.16129504 & 0.27466368 & 0.24097359 & 0.25014147 & 0.19975541\end{array}$


Figure 6: The effect of OM-85 and RV infection on Icam, INF- $\gamma$ and Myd88 expression.

A. ICAM expression was detected by Western-blotting (optical density)

Icam 1

\begin{tabular}{|c|c|c|c|c|c|c|c|c|}
\hline $\begin{array}{l}\text { control } \\
\text { control }\end{array}$ & day 0 & RV day 2 & $\begin{array}{l}\text { OM } 10 \\
\mathrm{ug} / \mathrm{ml}\end{array}$ & RV + OM 10 ug & $\begin{array}{l}\text { control } \\
\text { control }\end{array}$ & day 0 & RV day 2 & $\begin{array}{l}\text { OM } 10 \\
\mathrm{ug} / \mathrm{ml}\end{array}$ \\
\hline h1 & 184297 & 657495 & 160143 & 352424 & h1 & 1.125258 & 4.014458 & 0.977783 \\
\hline h2 & 183304 & 534258 & 145419 & 214231 & h2 & 1.119198 & 3.262011 & 0.887883 \\
\hline h3 & 160143 & 382758 & 243689 & 319285 & h3 & 0.977783 & 2.337 & 1.487887 \\
\hline h4 & 128374 & 490852 & 268835 & 474308 & h4 & 0.783811 & 2.996985 & 1.641423 \\
\hline h5 & 166430 & 315243 & 117564 & 201764 & h5 & 1.016167 & 1.924775 & 0.717809 \\
\hline h6 & 160143 & 553452 & 164444 & 775072 & h6 & 0.977783 & 3.379204 & 1.004046 \\
\hline \multirow[t]{2}{*}{ mean } & 163781.8 & & & & mean & 1 & 2.985739 & 1.119472 \\
\hline & & & & & sem & 0.072057 & 0.434821 & 0.209209 \\
\hline
\end{tabular}

asthma

$\begin{array}{lrlll}\text { a1 } & 139351 & 832819 & 159710 & 277691 \\ \text { a2 } & 127465 & 584719 & 236088 & 415904 \\ \text { a3 } & 118613 & 375313 & 276426 & 402879 \\ \text { a4 } & 125315 & 275162 & 125947 & 161228 \\ \text { a5 } & 148582 & 395419 & 160975 & 236341 \\ \text { a6 } & 167930 & 388084 & 312971 & 276426 \\ \text { a7 } & 126959 & 859627 & 123671 & 554496 \\ \text { mean } & 136316.3 & & & \end{array}$

asthma

$\begin{array}{lrrr}\text { a1 } & 0.850835 & 5.084934 & 0.97514 \\ \text { a2 } & 0.778259 & 3.570108 & 1.441478 \\ \text { a3 } & 0.724213 & 2.29154 & 1.687772 \\ \text { a4 } & 0.765133 & 1.680051 & 0.768994 \\ \text { a5 } & 0.907197 & 2.414301 & 0.982861 \\ \text { a6 } & 1.025325 & 2.36952 & 1.910904 \\ \text { a7 } & 0.775171 & 5.248615 & 0.755096 \\ \text { mean } & 0.832305 & 3.23701 & 1.217463 \\ \text { sem } & 0.060365 & 0.827303 & 0.26676\end{array}$

$\begin{array}{lrlrl}\text { COPD } & & & & \\ \text { c1 } & 217264 & 663520 & 95553 & 447612 \\ \text { c2 } & 129679 & 446390 & 165333 & 325573 \\ \text { c3 } & 228347 & 558548 & 101462 & 223704 \\ \text { c4 } & 298674 & 334742 & 227164 & 312645 \\ \text { c5 } & 134875 & 223806 & 124178 & 142537 \\ \text { c6 } & 119594 & 335264 & 222686 & 285764 \\ \text { c7 } & 266453 & 671928 & 129781 & 221667 \\ \text { c8 } & 96572 & 355013 & 137727 & 314253 \\ \text { mean } & 186432 & & & \end{array}$

COPD

$\begin{array}{lrrr}\text { c1 } & 1.326546 & 4.051244 & 0.583417 \\ \text { c2 } & 0.791781 & 2.725517 & 1.009474 \\ \text { c3 } & 1.394215 & 3.410317 & 0.619492 \\ \text { c4 } & 1.823609 & 2.043826 & 1.386992 \\ \text { c5 } & 0.823502 & 1.36649 & 0.758194 \\ \text { c6 } & 0.730205 & 2.047016 & 1.359648 \\ \text { c7 } & 1.626878 & 4.10258 & 0.792403 \\ \text { c8 } & 0.589637 & 2.167601 & 0.840917 \\ \text { mean } & 1.138297 & 2.739324 & 0.918817 \\ \text { sd } & 0.462298 & 1.014507 & 0.309764 \\ \text { sem } & 0.266908 & 0.585726 & 0.178842\end{array}$

B. He role of intracellular signalling on ICAM expression

\begin{tabular}{lcccccc} 
& & \multicolumn{2}{c}{$\mathrm{RV}+$} & $\mathrm{RV}+$ & $\mathrm{RV} 1+$ & $\mathrm{R}+$ \\
Cell lines & Control & RV & OM & OM+DDA & OM+SB & OM+PD \\
H01 & 1.174 & 4.386 & 2.968 & 4.233 & 1.978 & 3.879 \\
H02 & 0.886 & 3.975 & 3.175 & 3.996 & 2.735 & 4.285 \\
H03 & 1.047 & 4.823 & 3.867 & 4.175 & 2.186 & 3.576
\end{tabular}




$\begin{array}{lcccccc}\text { H04 } & 0.978 & 3.968 & 1.685 & 2.286 & 3.296 & 3.773 \\ \text { H05 } & 0.737 & 2.175 & 1.953 & 3.004 & 3.191 & 2.396 \\ \text { H06 } & 1.006 & 4.396 & 2.504 & 4.867 & 2.968 & 3.818 \\ \text { mean } & 0.97 & 3.95 & 2.69 & 3.76 & 2.73 & 3.62 \\ \text { SEM } & 0.06640462 & 0.41485548 & 0.3625247 & 0.42053827 & 0.28789045 & 0.28789045\end{array}$

\section{Interferon- $\gamma(\mathrm{pg} / \mathrm{ml} / \mathbf{2 4}$ hours) determined by ELISA}

Date/Time $\quad 08 / 29 / 2017$ 07:39:35

Quadratic Fit: $\mathrm{Y}=\mathrm{A}+\mathrm{BX}+\mathrm{CX^{ \wedge } 2}$

20/50/80\%: $X=99.458 / 279.762 / 529.291 \quad Y=0.476 / 1.111 / 1.746$

A: $0.063(+/-0.033), B: 0.004(+/-0.000), C:-0.000(+/-0.000)$

chi2 $=0.060, \mathrm{RMS}=0.074, \mathrm{r}^{\wedge} 2=0.992$

Standards Report:

\begin{tabular}{|c|c|c|c|c|c|c|c|}
\hline \multirow[t]{2}{*}{ Std \# } & \multicolumn{2}{|c|}{ Conc } & \multirow{2}{*}{$\begin{array}{l}\text { Well } \\
\text { G2 }\end{array}$} & \multirow{2}{*}{$\begin{array}{r}\text { Replicates } \\
0.049\end{array}$} & \multirow{2}{*}{$\begin{array}{l}\text { Mean } \\
0.053\end{array}$} & \multicolumn{2}{|r|}{$\% \mathrm{CV}$} \\
\hline & 1 & 15 & & & & 0.006 & 10.673 \\
\hline & & & $\mathrm{H} 2$ & 0.057 & & & \\
\hline & 2 & 31 & E2 & 0.21 & 0.2 & 0.013 & 6.701 \\
\hline & & & $\mathrm{F} 2$ & 0.191 & & & \\
\hline & 3 & 62 & $\mathrm{C} 2$ & 0.472 & 0.464 & 0.011 & 2.438 \\
\hline & & & D2 & 0.456 & & & \\
\hline & 4 & 125 & $\mathrm{~A} 2$ & 0.528 & 0.538 & 0.013 & 2.5 \\
\hline & & & B2 & 0.547 & & & \\
\hline & 5 & 250 & G1 & 0.96 & 0.962 & 0.002 & 0.221 \\
\hline & & & $\mathrm{H} 1$ & 0.963 & & & \\
\hline & 6 & 500 & E1 & 1.738 & 1.716 & 0.031 & 1.813 \\
\hline & & & $\mathrm{F} 1$ & 1.694 & & & \\
\hline & 7 & 1000 & $\mathrm{C} 1$ & 2.174 & 2.17 & 0.006 & 0.293 \\
\hline
\end{tabular}

Sample Report:

Sample ID Well

A1-C E9

A1-OM G9

A1-RV F9

A1-RV-OM H9

A2-RV-OM D10

A2-C A10

A2-OM C10

A2-RV B10

A3-C E10

A3-OM G10

A3-RV F10

A3-RV-OM H10

A4-C A11

A4-OM C11

A4-RV B11

A4-RV-OM D11

\begin{tabular}{|c|c|c|}
\hline Replicates & Mean & Conc \\
\hline 0.066 & 0.066 & $0.728 \quad(*)$ \\
\hline 0.384 & 0.384 & $76.291 \quad(*)$ \\
\hline 0.193 & 0.193 & $30.172(*)$ \\
\hline 0.539 & 0.539 & $115.557\left(^{*}\right)$ \\
\hline 0.417 & 0.417 & $84.504(*)$ \\
\hline 0.06 & 0.06 & $(*)$ \\
\hline 0.101 & 0.101 & $8.752\left({ }^{*}\right)$ \\
\hline 0.108 & 0.108 & $10.365(*)$ \\
\hline 0.081 & 0.081 & $4.159(*)$ \\
\hline 0.088 & 0.088 & $5.764(*)$ \\
\hline 0.145 & 0.145 & $18.936(*)$ \\
\hline 0.477 & 0.477 & $99.636(*)$ \\
\hline 0.03 & 0.03 & $(*)$ \\
\hline 0.273 & 0.273 & $49.206(*)$ \\
\hline 0.085 & 0.085 & $5.076(*)$ \\
\hline 0.298 & 0.298 & $55.236(*)$ \\
\hline
\end{tabular}




\begin{tabular}{|c|c|c|c|c|c|}
\hline A5-C & E11 & 0.175 & 0.175 & 25.943 & $(*)$ \\
\hline A5-OM & G11 & 0.374 & 0.374 & 73.817 & $(*)$ \\
\hline A5-RV & F11 & 0.188 & 0.188 & 28.995 & $(*)$ \\
\hline A5-RV-OM & $\mathrm{H} 11$ & 0.485 & 0.485 & 101.674 & $(*)$ \\
\hline$A 6-C$ & A12 & 0.04 & 0.04 & $(-)$ & $(*)$ \\
\hline A6-OM & $\mathrm{C} 12$ & 0.109 & 0.109 & 10.596 & $(*)$ \\
\hline A6-RV & B12 & 0.078 & 0.078 & 3.472 & $(*)$ \\
\hline A6-RV-OM & $\mathrm{D} 12$ & 0.278 & 0.278 & 50.409 & $(*)$ \\
\hline A7-C & $\mathrm{E} 12$ & 0.094 & 0.094 & 7.142 & $(*)$ \\
\hline A7-OM & G12 & 0.189 & 0.189 & 29.231 & $(*)$ \\
\hline A7-RV & F12 & 0.211 & 0.211 & 34.421 & $(*)$ \\
\hline A7-RV-OM & $\mathrm{H} 12$ & 0.526 & 0.526 & 112.194 & $(*)$ \\
\hline C1-C & A6 & 0.027 & 0.027 & $(-)$ & $(*)$ \\
\hline C1-OM & C6 & 0.094 & 0.094 & 7.142 & $(*)$ \\
\hline C1-RV & B6 & 0.078 & 0.078 & 3.472 & $(*)$ \\
\hline C1-RV-OM & D6 & 0.311 & 0.311 & 58.387 & $(*)$ \\
\hline $\mathrm{C} 2-\mathrm{C}$ & E6 & 0.029 & 0.029 & $(-)$ & $(*)$ \\
\hline C2-OM & G6 & 0.211 & 0.211 & 34.421 & $(*)$ \\
\hline C2-RV & F6 & 0.069 & 0.069 & 1.413 & $(*)$ \\
\hline C2-RV-OM & $\mathrm{H} 6$ & 0.738 & 0.738 & 168.806 & $(*)$ \\
\hline C3-C & A7 & 0.316 & 0.316 & 59.602 & $(*)$ \\
\hline C3-OM & $\mathrm{C} 7$ & 0.177 & 0.177 & 26.412 & $(*)$ \\
\hline C3-RV & B7 & 0.166 & 0.166 & 23.835 & $(*)$ \\
\hline C3-RV-OM & D7 & 0.738 & 0.738 & 168.806 & $(*)$ \\
\hline $\mathrm{C} 4-\mathrm{C}$ & E7 & 0.118 & 0.118 & 12.674 & $(*)$ \\
\hline C4-OM & G7 & 0.145 & 0.145 & 18.936 & $(*)$ \\
\hline C4-RV & F7 & 0.167 & 0.167 & 24.069 & $(*)$ \\
\hline C4-RV-OM & $\mathrm{H} 7$ & 0.434 & 0.434 & 88.765 & $(*)$ \\
\hline C5-C & A8 & 0.263 & 0.263 & 46.805 & $(*)$ \\
\hline C5-OM & C8 & 0.383 & 0.383 & 76.043 & $(*)$ \\
\hline C5-RV & B8 & 0.288 & 0.288 & 52.819 & $(*)$ \\
\hline C5-RV-OM & D8 & 0.998 & 0.998 & 244.287 & $(*)$ \\
\hline C6-C & E8 & 0.077 & 0.077 & 3.243 & $(*)$ \\
\hline C6-OM & G8 & 0.176 & 0.176 & 26.177 & $(*)$ \\
\hline C6-RV & F8 & 0.041 & 0.041 & $(-)$ & $(*)$ \\
\hline C6-RV-OM & $\mathrm{H} 8$ & 0.384 & 0.384 & 76.291 & $(*)$ \\
\hline C7-C & A9 & 0.188 & 0.188 & 28.995 & $(*)$ \\
\hline C7-OM & $\mathrm{Cg}$ & 0.263 & 0.263 & 46.805 & $(*)$ \\
\hline C7-RV & B9 & 0.094 & 0.094 & 7.142 & $(*)$ \\
\hline C7-RV-OM & D9 & 0.665 & 0.665 & 148.868 & $(*)$ \\
\hline $\mathrm{H} 1-\mathrm{C}$ & A3 & 0.039 & 0.039 & $(-)$ & $(*)$ \\
\hline H1-OM & C3 & 0.184 & 0.184 & 28.055 & $(*)$ \\
\hline H1-OM-RV & D3 & 0.637 & 0.637 & 141.349 & $(*)$ \\
\hline $\mathrm{H} 1-\mathrm{RV}$ & B3 & 0.176 & 0.176 & 26.177 & $(*)$ \\
\hline $\mathrm{H} 2-\mathrm{C}$ & E3 & 0.059 & 0.059 & $(-)$ & $(*)$ \\
\hline $\mathrm{H} 2-\mathrm{OM}$ & G3 & 0.265 & 0.265 & 47.285 & $(*)$ \\
\hline $\mathrm{H} 2-\mathrm{RV}$ & F3 & 0.095 & 0.095 & 7.372 & $(*)$ \\
\hline
\end{tabular}




$\begin{array}{llllrl}\text { H2-RV-OM } & \text { H3 } & 0.639 & 0.639 & 141.884(*) \\ \text { H3-C } & \text { A4 } & 0.051 & 0.051(-) & (*) \\ \text { H3-OM } & \text { C4 } & 0.364 & 0.364 & 71.35(*) \\ \text { H3-RV } & \text { B4 } & 0.202 & 0.202 & 32.294(*) \\ \text { H3-RV-OM } & \text { D4 } & 0.738 & 0.738 & 168.806(*) \\ \text { H4-C } & \text { E4 } & 0.105 & 0.105 & 9.673(*) \\ \text { H4-OM } & \text { G4 } & 0.189 & 0.189 & 29.231(*) \\ \text { H4-RV } & \text { F4 } & 0.208 & 0.208 & 33.711(*) \\ \text { H4-RV-OM } & \text { H4 } & 0.531 & 0.531 & 113.486(*) \\ \text { H5-C } & \text { A5 } & 0.045 & 0.045(-) & (*) \\ \text { H5-OM } & \text { C5 } & 0.134 & 0.134 & 16.38(*) \\ \text { H5-RV } & \text { B5 } & 0.057 & 0.057(-) & (*) \\ \text { H5-RV-OM } & \text { D5 } & 0.656 & 0.656 & 146.444(*) \\ \text { H6-C } & \text { E5 } & 0.089 & 0.089 & 5.993(*) \\ \text { H6-OM } & \text { G5 } & 0.374 & 0.374 & 73.817(*) \\ \text { H6-RV } & \text { F5 } & 0.134 & 0.134 & 16.38(*) \\ \text { H6-RV-OM } & \text { H5 } & 0.407 & 0.407 & 82.007(*)\end{array}$

Analysis:

$\begin{array}{ccccc}\text { Healthy } & \text { untreated } & \text { RV } & \text { OM } & \text { RV+OM } \\ \text { H1 } & 0 & 26.177 & 28.055 & 141.349 \\ \text { H2 } & 0 & 7.372 & 47.285 & 141.884 \\ \text { H3 } & 0 & 32.294 & 71.35 & 168.806 \\ \text { H4 } & 9.673 & 33.711 & 29.231 & 113.486 \\ \text { H5 } & 0 & 0 & 16.38 & 146.444 \\ \text { H6 } & 5.993 & 16.38 & 73.817 & 82.007 \\ \text { Mean } & 2.611 & 19.3223333 & 44.353 & 132.329333 \\ \text { SEM } & 1.8823289 & 6.15501317 & 10.737505 & 13.5496502\end{array}$

Asthma

$\begin{array}{lcccc}\text { A1 } & 0.728 & 30.172 & 76.291 & 115.557 \\ \text { A2 } & 0 & 10.365 & 8.752 & 84.504 \\ \text { A3 } & 4.159 & 18.936 & 5.764 & 99.636 \\ \text { A4 } & 0 & 5.076 & 49.206 & 55.236 \\ \text { A5 } & 25.943 & 28.995 & 73.817 & 101.674 \\ \text { A6 } & 0 & 3.472 & 10.596 & 50.409 \\ \text { A7 } & 7.143 & 24.421 & 29.231 & 112.194\end{array}$

$\begin{array}{lllll}\text { Mean } & 5.42471429 & 17.3481429 & 36.2367143 & 88.4585714\end{array}$

$\begin{array}{llllll}\text { SEM } & 3.85823897 & 4.54778428 & 12.444709 & 10.7553935\end{array}$

COPD

$\begin{array}{lcccc}\text { C1 } & 0 & 3.472 & 7.142 & 58.387 \\ \text { C2 } & 0 & 1.413 & 34.421 & 168.806 \\ \text { C3 } & 59.6 .2 & 23.835 & 26.412 & 168.806 \\ \text { C4 } & 12.674 & 24.069 & 18.936 & 88.765 \\ \text { C5 } & 46.805 & 52.819 & 76.043 & 244.287\end{array}$




$\begin{array}{ccccc}\text { C6 } & 3.243 & 0 & 26.177 & 76.291 \\ \text { C7 } & 28.995 & 7.142 & 46.805 & 148.868 \\ \text { Mean } & 15.2861667 & 16.1071429 & 33.7051429 & 136.315714 \\ \text { SEM } & 7.74718019 & 7.79289189 & 9.12597615 & 26.8035637\end{array}$

D. MyD88 expression and modification was determined by an in house developed ELISA

\begin{tabular}{lrrrrr} 
Myd88 & CONTROLS & \multicolumn{2}{c}{ 48 hrs } \\
control & \multicolumn{2}{c}{ 24 hrs } \\
control & \multicolumn{1}{c}{ day0 } & OM 10 & \\
h1 & 165473 & 184297 & 160143 & 198574 & 203752 \\
h2 & 142537 & 132464 & 149968 & 176453 & 214231 \\
h3 & 156342 & 160143 & 176453 & 143527 & 177564 \\
h4 & 114967 & 128374 & 142561 & 142364 & 154928 \\
h5 & 152436 & 166430 & 164735 & 198672 & 186252 \\
h6 & 169584 & 160143 & 164444 & 217684 & 221761 \\
mean & 150223.167 & 155308.412 & 159717.399 & 179545.667 & 193081.333
\end{tabular}

Analysis controls: ratio day 0 / 24 / 48 hrs

\begin{tabular}{lrrrr}
\multicolumn{1}{l}{ 24 hrs } & \multicolumn{4}{l}{48 hrs } \\
$\begin{array}{l}\text { control } \\
\text { control }\end{array}$ & \multicolumn{1}{l}{ control 24 } & \multicolumn{1}{l}{ ug/ml } & control 48 & OM 10 ug \\
h1 & 111.375764 & 96.7789404 & 120.003868 & 123.133079 \\
h2 & 92.933063 & 105.213383 & 123.794524 & 150.298519 \\
h3 & 102.431219 & 112.863466 & 91.8032263 & 113.574088 \\
h4 & 111.661607 & 124.00167 & 123.830317 & 134.75867 \\
h5 & 109.179998 & 108.068304 & 130.331418 & 122.183736 \\
h6 & 94.4328569 & 96.9692766 & 128.363525 & 130.767643 \\
mean & 103.669085 & 107.31584 & 119.687813 & 129.119289 \\
sem & 4.86994777 & 5.95682932 & 8.1659038 & 7.34243084
\end{tabular}

COPD

\begin{tabular}{lrrrrr} 
& \multicolumn{3}{c}{ 24 hrs } & 48 hrs \\
I & & control 24 & $\begin{array}{r}\text { OM 10 } \\
\text { ug/ml }\end{array}$ & control 48 & OM 10 ug \\
I & day0 & & & & \\
c1 & 198675 & 217264 & 195553 & 210697 & 226175 \\
c2 & 110895 & 109679 & 165333 & 142365 & 152438 \\
c3 & 216643 & 228347 & 201462 & 238107 & 217564 \\
c4 & 178539 & 198674 & 127164 & 197236 & 210985 \\
c5 & 110978 & 114875 & 124178 & 132978 & 148275 \\
c6 & 109685 & 129594 & 142686 & 123351 & 130075
\end{tabular}




$\begin{array}{llrlll}\text { c7 } & 164739 & 176453 & 129781 & 168664 & 187564 \\ \text { c8 } & 109786 & 96572 & 137727 & 126328 & 132467 \\ \text { mean } & 149993 & 158932 & 152985 & 167466 & 175693\end{array}$

Analysis COPD: ratio day 0 / 24 / 48 hrs

\begin{tabular}{|c|c|c|c|c|}
\hline & $24 \mathrm{hrs}$ & \multicolumn{3}{|c|}{$48 \mathrm{hrs}$} \\
\hline & control 24 & $\begin{array}{l}\text { OM } 10 \\
\mathrm{ug} / \mathrm{ml}\end{array}$ & control 48 & OM 10 ug \\
\hline c4 & 111.277648 & 71.2247744 & 110.472222 & 118.17306 \\
\hline c5 & 103.511107 & 111.89453 & 119.823749 & 133.607562 \\
\hline$c 6$ & 118.151257 & 130.086734 & 112.459315 & 118.589597 \\
\hline c7 & 107.110642 & 78.7798311 & 102.382557 & 113.85525 \\
\hline c8 & 87.9636857 & 125.45032 & 115.067495 & 120.659283 \\
\hline mean & 105.209618 & 107.243408 & 113.067771 & 119.576642 \\
\hline sem & 5.18801988 & 15.4525235 & 4.70335748 & 6.7374506 \\
\hline
\end{tabular}

\section{Asthma}

\begin{tabular}{lrrrrr} 
& \multicolumn{5}{c}{24 hrs } \\
I & day0 & control 24 & ug $/ \mathrm{ml}$ & control 48 & OM 10 ug \\
I & 117236 & 120967 & 138376 & 126385 & 137691 \\
a1 & 132454 & 127465 & 142309 & 140680 & 135904 \\
a2 & 109685 & 118613 & 139935 & 118734 & 142879 \\
a3 & 117587 & 117163 & 125947 & 124231 & 121228 \\
a4 & 130895 & 139286 & 145398 & 129786 & 136341 \\
a5 & 164538 & 167930 & 153648 & 170065 & 176426 \\
a6 & 110986 & 126959 & 134256 & 112453 & 154496 \\
a7 & 126197.286 & 131197.419 & 139981.313 & 131762 & 143566.408
\end{tabular}

Analysis asthma: ratio day 0 / 24 / 48 hrs

\begin{tabular}{|c|c|c|c|c|}
\hline & $24 \mathrm{hrs}$ & \multicolumn{3}{|c|}{$48 \mathrm{hrs}$} \\
\hline & control 24 & $\begin{array}{l}\text { OM } 10 \\
\mathrm{ug} / \mathrm{ml}\end{array}$ & control 48 & OM 10 ug \\
\hline a3 & 108.139594 & 127.578976 & 108.249989 & 130.263261 \\
\hline a4 & 99.6394159 & 107.109789 & 105.650284 & 103.096069 \\
\hline a5 & 106.410482 & 111.079873 & 99.152756 & 104.160325 \\
\hline a6 & 102.061277 & 93.3814681 & 103.359102 & 107.225236 \\
\hline a7 & 114.391736 & 120.966608 & 101.321788 & 139.203508 \\
\hline mean & 104.294014 & 112.227005 & 104.535471 & 114.857221 \\
\hline em & 3.44879821 & 6.46069367 & 1.96092367 & 8.49156211 \\
\hline
\end{tabular}

